\title{
Using Syndromic Surveillance Data to Monitor Endocarditis and Sepsis among Drug Users
}

\author{
Lana Deyneka*1, Anne Hakenewerth1, Zachary Faigen', Amy Ising ${ }^{2}$ and Clifton Barnett ${ }^{2}$ \\ ${ }^{1}$ Epidemiology, NCDPH, Raleigh, NC, USA; ${ }^{2}$ UNC, Chapel hill, NC, USA
}

\section{Objective}

To describe how the state syndromic surveillance system (NC DETECT) was used to initiate near real time surveillance for endocarditis, sepsis and skin infection among drug users.

\section{Introduction}

Recreational drug use is a major problem in the United States and around the world. Specifically, drug abuse results in heavy use of emergency department (ED) services, and is a high financial burden to society and to the hospitals due to chronic ill health and multiple injection drug use complications. Intravenous drug users are at high risk of developing sepsis and endocarditis due to the use of a dirty or infected needle that is either shared with someone else or re-used. It can also occur when a drug user repeatedly injects into an inflamed and infected site or due to the poor overall health of an injection drug user. The average cost of hospitalization for aortic valve replacement in USA is about $\$ 165,000$, and in order for the valve replacement to be successful, patients must abstain from using drugs.

\section{Methods}

We examined temporal trends of drug-related visits to hospital EDs, as well as drug-related related ED admissions complicated with endocarditis, bacteremia and sepsis.

\section{Results}

The trends in Endocarditis/Sepsis and drug-related related admissions appear to echo overdose related ED admissions increase. Patients ED return visits and hospitalizations for the same problem are also growing compare to the previous years. We will discuss the NC DETECT case definition used to monitor drug overdose/dependence and infection, case definition transition from ICD-9 to ICD-10 codes, and will share surveillance analysis results.

\section{Conclusions}

NC DETECT's system flexibility has been important in rapidly establishing surveillance of infections among drug users. Near real time analysis on hospital, county and state levels can be performed using NC DETECT system reports to provide state officials, hospitals and LHDs with situational awareness. Limitations: Syndromic surveillance ED data contains less accurate information about the diagnosis codes, procedures, length of stay, and severity comparing to the hospital discharge data.

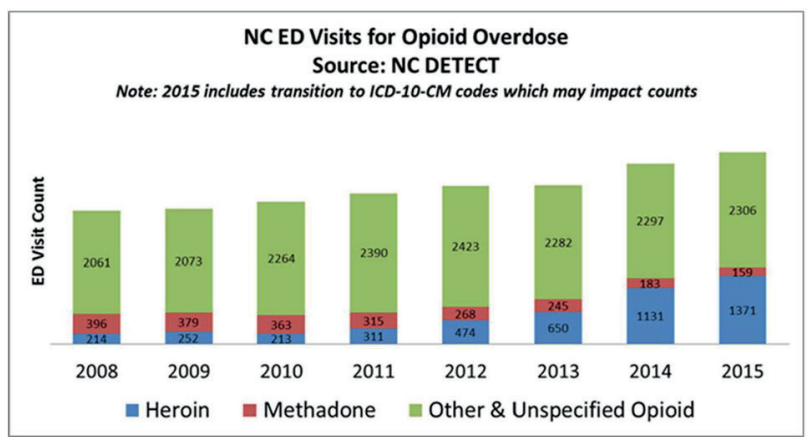

NC ED Visits for Drug Overdose Dependence and Endocarditis and/or Bacteremia/Sepsis

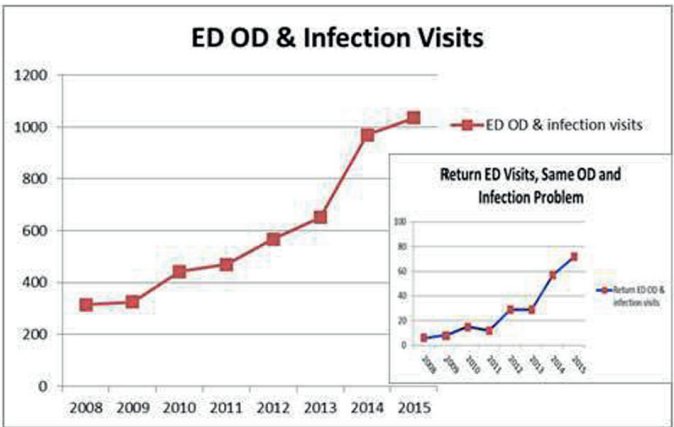

NC DETECT Drug Overdose / Dependence and Infection Syndrome

This case definition looks for ED visits with a diagnosis of medication OR drug overdose, dependence AND (Endocarditis OR Bacteremia/Sepsis OR Skin and soft tissue infection) Medication OR drug overdose / dependence

ICD-9-CM Codes: 292.0 OR 304.90 OR 305.90 OR E962.0 OR 960-979.9 OR E850E858.9 OR E950-E950.5 OR E980-E980.5 OR 304.0-304.02 OR 304.20-304.22 OR 304.40-304.42 OR 304.5-304.52 OR 304.60-304.62 OR 304.70-304.72 OR 304.80304.82

ICD-10-CM Codes: T36-T50 (ending in A or D only and EXCLUDING all codes in which the second to last character ( $7^{\text {th }}$ position) is a 5 (adverse effect) or 6 (underdosing)OR F19.939 OR F14.20 OR F15.20 OR F11.20 OR F16.20 OR F19.20 OR F19.230 OR F19.231 OR F19.232

Endocarditis

ICD-9-CM Codes: 421 OR R421.1 OR 421.9 OR 424.9

ICD-10-CM Codes: I33.0 OR I39 OR I33.9 OR I38

Bactermia/Sepsis

CD-9-CM Codes 790.7 OR 995.91 OR 995.92 OR 038-038.4 OR 038.8 OR 038.9 ICD-10-CM Codes: R78.81 OR A41.9 OR A40.9 OR A40.3 OR A41.4 OR A41.8

Skin and soft tissue infection

ICD-9-CM Codes: $686 \%$ OR 682.0-682.9

ICD-10-CM Codes: K12.2 OR L03.211 OR L03.212 OR L03.221 OR L03.222 OR L03.319 OR L03.329 OR L03.119 OR L03.129 OR L03.317 OR L03.811 OR L03.818 OR L03.891 OR L03.898 OR L03.90 OR L03.91 OR L08.0 OR L08.89 OR L08.9

\section{Keywords}

surveillance; drug users; endocarditis; bacteremia; sepsis

\section{References}

1. Ising A, Proescholdbell S, Harmon KJ, Sachdeva N, Marshall SW, Waller AE. Use of syndromic surveillance data to monitor poisonings and drug overdoses in state and local public health agencies. Injury prevention. 2016 Apr 1;22(Suppl 1):i43-9.

2. Jennifer A. Frontera Jeremy D. Gradon Right-Side Endocarditis in Injection Drug Users: Review of Proposed Mechanisms of Pathogenesis Oxford Journals Medicine \& Health Clinical Infectious Diseases, Volume 30, Issue 2

3. Hannah L. F. Cooper, Joanne E. Brady, Daniel Ciccarone, Barbara Tempalski1 Karla Gostnell, Samuel R. Friedman Increasing Infectious Endocarditis Admissions Among Young People Who Inject Drugs Open Forum Infect Dis (2016) 3 (3): ofw157 Accepted July 18, 2016

\section{*Lana Deyneka}

E-mail: lana.deyneka@dhhs.nc.gov 